Research Article

\title{
Caudal block with general anesthesia for outpatient pediatric surgery procedures
}

\begin{abstract}
Background: Pediatric surgery is mostly done in an outpatient setting and due to this fact preoperative and postoperative pain management is a very important task to overcome.
\end{abstract}

\begin{abstract}
Aim: We aim to share and underline our clinical experience regarding with the children's comfort and parents' satisfaction after outpatient pediatric surgery cases performed under general anesthesia combined with caudal block regional anesthesia.
\end{abstract}

Methods: The files of 405 ASA1 outpatient pediatric surgery patients were retrospectively reviewed. Each inguinoscrotal procedures performed under general anesthesia combined with caudal extradural $1 \mathrm{ml} / \mathrm{kg}$ bupivacaine. Caudal extradural block was performed just after general anesthesia induction just before the operation. The objective pain score was used to determine child analgesia requirement level postoperatively up to 12 hours. Postoperative pain control was sustained for 48 hours using routine paracetamol and ibuprofen if needed.

Results: Combined anesthesia and caudal block during outpatient procedures leads usage of low dose of inhalation anesthetic requirement and early recovery from the anesthetic condition. This was achieved by effective pain control of caudal block. The main concentration of caudal block performed according to the types of outpatient procedures. In hypospadias patient bupivacaine concentration were kept at high level aim to prolong lower extremities immobilization for better recovery. Combined anesthesia with caudal block for outpatient procedures made possible to discharge all most all of the patients as day case surgery.

Conclusion: Combined general anesthesia with caudal analgesia with bupivacaine enable us effective and safe per operative pain control beside usage of lower dose of inoperative anesthetic agents makes day case surgery more safer for children with less side effects.
Volume 7 Issue I - 2017

\author{
Irsi Cesim,' Ar Yıldırım Arzu, ${ }^{2}$ Ozdemir \\ Firdevs ${ }^{2}$ \\ 'Department of Pediatric Surgery, Istanbul Fatih Sultan Mehmet \\ Teaching and Research Hospital, Turkey \\ ${ }^{2}$ Anesthesiology and Reanimation, Istanbul Fatih Sultan Mehmet \\ Teaching and Research Hospital, Turkey
}

Correspondence: Cesim Irsi, Department of Pediatric Surgery, Istanbul Fatih Sultan Mehmet Teaching and Research Hospital, Turkey, Tel +90 532 2262072, Fax +90 2165750406 , Email csmirsi@yahoo.com

Received: October 27, 2016 | Published: January 09, 2017

\section{Introduction}

\section{What this study adds:}

\section{A. What is known about this subject?}

a. Pediatric surgery is mostly done in an outpatient setting and due to this fact preoperative and postoperative pain management is a very important task to overcome

b. Proper pain control preoperatively leads better recovery of child and also reduces unnecessary hospital admissions.

c. Lowering general anesthetic doses in operatively and effective postoperative pain control areremarkable benefits of caudal extradural analgesia especially for day-case procedures.

\section{B. What new information is offered in this study?}

Our findings and results of this study compared to other similar studies in the literature are same and we just want to mention and underline caudal block importance for day-case surgery performing clinics.

\section{What are the implications for research, policy, or practice?}

Pediatric surgeons and urologist should pay attention to pain managemet for outpatient surgery which enable better results for both parents and children.

\section{Background}

Postoperative pain control from pediatric surgical procedures is a great challenge to overcome due to family and children anxiety. Discomfort of the outpatient operation sometimes become more important task than operation itself because of unfamiliar and anxious parents and child. That is why proper pain control will be a very comfortable condition and less emotionally disturbing experience for patient and family, and also it reduces unnecessary hospital admissions. ${ }^{1}$

Sensation of pain and discomfort after an operation usually differ from patient to patient and this subjective measurement of the condition, sometimes cause unnecessary usage of analgesic drugs. Recent studies citing about improper and inadequate analgesia usage mostly indicate this subtle fact. ${ }^{2}$

Current pediatric surgery outpatient pain management strategies differ from clinics to clinics and they perform analgesia via parenterally, local or oral routes according to their clinical experience and preferences. Recently after growing realization of creating proper per operative pain control is very effective postoperatively; caudal block combined with general anesthesia become more popular 3-6. Combine usage of caudal block with general anesthesia beside effective pain control postoperatively enable us usage of lesser inhaler anesthesia agent and early recovery from inguinoscrotal surgery cases postoperatively. 


\section{Methods}

The data was obtained from files of consecutive 405 cases of outpatient pediatric patients at our hospital from March 2012 to December 2015. Data obtained were patient' personal details, diagnoses, procedures performed, modes of anesthesia, postoperative pain score and family satisfactions. The consent of the patients for operations was taken from all of families. ${ }^{10}$ Caudal block were applied to the all children by anesthesiologist. In the left lateral decubitus position with aseptic preparation of the skin, 22-gauge needle was inserted at a right angle position to the skin until it pierced the sacrococcygeal ligament and then into the sacral canal (Figure 1). One milliliter per kilogram 0.1 per cent bupivacaine for inguinoscrotal plus circumcision, per cent 1.5 bupivacaine for hypospadias and.$^{10}$ per cent for circumcision only were then injected in 45 second with vital monitoring and maximum volume was $20 \mathrm{ml}$. with standard.

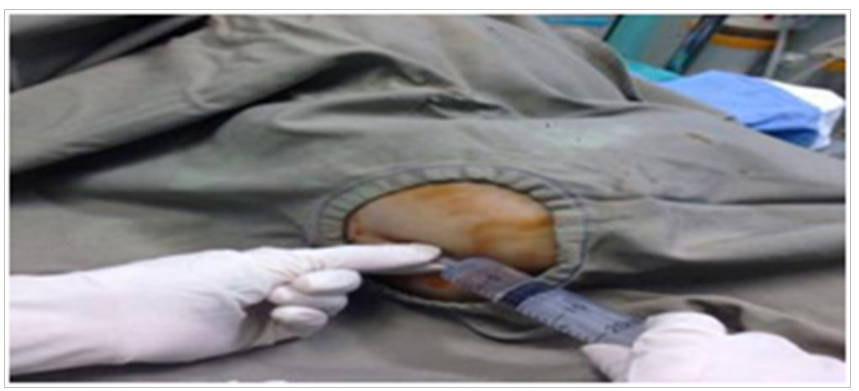

Figure I The pattern of caudal block application to the patient.

Local penile block were performed in case of caudal block performing insufficiency due to unfamiliar anesthesiologist. Otherwise in all outpatient inguinoscrotal pediatric surgery procedures caudal block were performed. Postoperatively, the patients are observed for about 4 to 6 hour. Postoperative pain and analgesic requirements were evaluated with Objective Pain scale (OPS) (from 1 to 5) or The WongBaker FACES pain score were used for assessing pain in children, especially if their communication is limited. Supplemental analgesia (acetaminophen or ibuprofen) in case of need were used when evidence of pain exist. Bromage scale $(0=$ no motor block, $1=$ can move legs, $2=$ cannot move legs) were used to help the nurse in charge to assess the patient's condition when the child is unable to explain or has difficulty describing what is happening to him or her. Any side effects were noted. If analgesic was needed at home (OPS 4-5), oral 30-40 mg per kg paracetamol was suggested.

\section{Results}

A total of 405 outpatient pediatric operations were performed during 4-year study period. The patients were aged from 1 month to 17 years with a mean age of 6.5 years. While 65 (16.2 per cent) were females and 340 ( 83.8 per cent) were males. Caudal block anesthesia within combination to general anesthesia was the only form of anesthesia administered, mainly for the inguinoscrotal surgery and circumcision procedures. Local anesthesia in the forms of local infiltration was not included in the presented study population. The procedures carried out are summarized in Table 1. Combined anesthesia and caudal block during outpatient procedures leads usage of low dose of inhalation anesthesia requirement and early recovery from the anesthesia. This was achieved by effective pain control of caudal block. The main concentration of caudal block performed according to the types of outpatient procedures. In hypospadias patient's bupivacaine concentration were kept at high level aim to prolong lower extremity immobilization for better recovery. As shown with in the Table 1, lower concentration of bupivacaine was given to the circumcision cases.

Table I Frequency of outpatient pediatric Surgery procedures

\begin{tabular}{lll}
\hline Types of procedures & $\mathbf{N}: 405$ & Bupivacaine concentration (\%)* \\
\hline Herniorrhaphy & 125 & $0.10 \%$ \\
Hydrocelectomy & 40 & $0.10 \%$ \\
Undescended testis & 35 & $0.10 \%$ \\
Hypospadias & 25 & $1.50 \%$ \\
Circumcision & 180 & $0.25 \%$ \\
\hline
\end{tabular}

*Bupivacaine at the standard dose of Icc per kg per patient.

Five criteria which already classified according to the OPS or The Wong-Baker faces pain score. ${ }^{1}$ crying, agitation, moves, posture and localization of place of pain were assessed postoperatively. ${ }^{1}$ Total score was between 0 - 10 and each criterion was given points between 0-2. Patients having OPS 4-6 were given oral or rectal $30-40 \mathrm{mg} /$ $\mathrm{kg}$ paracetamol. Analgesia requirement among 405 consecutive postoperative outpatients whose most of them tolerate the procedures very well were only 25 patient and they required extra dose of analgesia per oral or intravenous paracetamol / ibuprofen. Globe vesicale was observed at the beginning of study between 6 children and catheter drainage was performed. This was overcomed by rearrangement of caudal block bupivacaine concentration as shown in the Table 1.

Postoperative patients comfort was very remarkable and this leads high parent's satisfaction. A calm painless postoperative patient and happy parents also lead a good comfortable working situation also for pediatric surgery staff and mostly for nurses in charge. All postoperative 405 patients including hypospadias ones were discharged on the same day as day case surgery and this was achieved mostly by combined general and caudal block analgesia. After 1 week of the surgery, we order to family to come back for control and at that time there was no any prominent discomfort or pain related complaint defined by the parents.

\section{Discussion}

Postoperative pain control is a very important part of surgical procedures because of very sensitive children and anxious parents and also it may play a role in the postoperative healing process. That's why many different pain control modalities have been performed and many articles have done to compare their effectiveness. Regional anesthetic techniques like caudal block have thought proper for pediatric patients. ${ }^{4,5}$ Lowering general anesthetic requirements intraoperatively and providing adequate postoperative pain relief are well known two major benefits of regional supplementation especially for day-case procedures.

Although it is not clear but some researchers believe in that improper usage of inhalation anesthesia agents in a still neurologically developing child patient may adversely affect their nervous system. Similar to this condition there is another disquiet condition to analgesic agent usage for per operative pain relief. Caudal block effective usage with better results will resolve these problems. ${ }^{6,7}$

Bupivacaine is a well known local anesthesia agent with long duration of action and prominent sensory motor block. Also it is a very common and indispensable component of caudal block because of these features. ${ }^{8}$ But for better postoperative pain management due to relatively short duration of action of bupivacaine is a better and a long acting new agent and adjuvant that have been investigated. ${ }^{9} \mathrm{~A}$ better long acting caudal block usable agent for researches is still a 
hot topics. We observed an early anesthesia recovery of patient from the operations and a better postoperative pain control with the usage of caudal bupivacaine dose related block combined with general anesthesia in outpatient pediatric surgery case This observation seems regular with the pediatric anesthesia literature. There is a trend toward usage of non-opioids, non-steroidal agent as an analgesic agent because of fewer side effects. Nowadays intravascular acetaminophen is one of them and in our clinic we use it confidently in case of parenteral analgesia requirement. ${ }^{10,11}$

The most important obstacle of outpatient surgery is a agitated, painful child beside with symptoms like nausea, vomiting which are not rare side effects of general anesthesia. Caudal block combined with general anesthesia enable pediatric surgeon and pediatric urologist to perform day case surgery more effectively. ${ }^{12,13}$ Because it decreases per operative inhalation anesthetic doses, and provide better postoperative pain control. Relatively sensitive inguinoscrotal cases like hypospadias have been performed as day case surgery by effective combination of caudal block with general anesthesia. In our clinic distal hypospadias cases with stent or without stent have undergone day case surgery without prominent complications. With our experience we observed only 9 cases of globe vesicales as a complications of caudal analgesia and this problem was overcome by reduction of bupivacaine concentration.

In the literature, there are several resarches comparing effective analgesia technique for hypospadias surgery. ${ }^{14,15}$ Our experience with hypospadias with or without stent are similar with other same literature researches regarding better results with caudal combined general anesthesia comparing with other modalities like penile block or parenteral analgesia. This study confirm that pediatric outpatient surgery is feasible and effective in this setting by combined usage of caudal and general anesthesia. It would significantly help to reduce waiting time for outpatient surgery and pressure on hospital beds. Also it reduces unnecessary hospital admissions and very useful for clinics which are very busy.

\section{Conclusion}

Management of surgical pain is a very important topic to overcome in pediatric patient recovery both for a calm painless patient healing process, satisfied and better coherent parents for child treatment. Effective combine caudal block with general anesthesia make outpatient surgery more feasible and can prevent unnecessary hospital admissions.

\section{Ethics committee approval}

The name of committee is Istanbul Fatih Sultan Mehmet Teaching and Research Hospital Ethics Committe; and approval reference number: 2015/159753.

\section{Conflicts of interest}

There is no conflict of interest.

\section{Acknowledgements}

None.

\section{Funding}

None.

\section{References}

1. Shanthanna H, Singh B, Guyatt G. A Systematic review and metaanalysis of caudal block as compared to non-caudal regional techniques for inguinal surgeries in children. Biomed Res Int. 2014:890626.

2. de Beer DA, Thomas ML. Caudal additives in children - solutions or problem? Br J Anaesth. 2003;90(4):487-498.

3. The association of Paediatric anaesthetists of Great Britain and Ireland Good Practice in Postoperative and Procedural Pain Management, 2nd edition. Pediatr Anaesth. 2012;22 Suppl 1:1-79.

4. Sasaoka N, Kawaguchi M, Yoshitani K, et al. Evaluation of genitofemoral nerve block, in addition to ilioinguinal and iliohypogastric nerve block, during inguinal hernia repair in children. Br J Anaesth. 2005;94(2):243246

5. Fell D, Derrington C, Taylor E, et al. Paediatric postoperative analgesia, a comparison between caudal block and wound infiltration of local anaesthetic. Anaesthesia. 1988;43(2):107-110.

6. Dalens B, Snaoui A. Caudal Anaesthesia in Pediatric Surgery success rate and adverse effects in 750 consecutive patients. Anesth Analg. $1989 ; 68(2): 83-89$

7. Morgan GE, Mikhail MS. Pediatric anesthesia. In: Morgan GE, Mikhail MS, editors. Clinical Anesthesiology. 2nd (edn), Appleton and Lange, USA. 1986:726-742.

8. Gunter JB, Dunn CM, Bennie JB, et al. Optimum concentration of bupivacaine for combined caudal - General anesthesia in children. Anesthesiology. 1991;75(1):57-61.

9. Farrag WS, Ibrahim AS, Mostafa MG, et al. Ketamine versus magnesium sulfate with caudal bupivacaine block in pediatric inguinoscrotal surgery: A prospective randomized observer-blinded study. Urol Ann. 2015;7(3):325-329.

10. Stewart DW, Ragg PG, Sheppard S, et al. The severity and duration of postoperative pain and analgesia requirements in children after tonsillectomy, orchiopexy, or inguinal hernia repair. Paediatr Anaesth. 2012;22(2):136-143.

11. Morrison K, Herbst $\mathrm{K}$, Corbett $\mathrm{S}$, et al. Pain management practice patterns for common pediatric urology procedures. Urology. 2014;83(1):206-210.

12. Samuel M, Hampson-Evans D, Cunnington P. Prospective to randomized double-blind controlled trial to assess efficacy of double caudal analgesia in hypospadias repair. J Pediatr Surg. 2002;37(2):168174

13. Thies KC, Driessen J, Kho HG. Longer than expected duration of caudal analgesia with two different doses of levobupivacaine in children undergoing hypospadias repair. J Pediatr Urol. 2010;6(6):585-588.

14. Kundra P, Yuvaraj K, Agrawal K. Surgical outcome in children undergoing hypospadias repair under caudal epidural vs penile block. Paediatr Anaesth. 2012;22(7):707-712.

15. Seyedhejazi M, Azerfarin R, Kazemi F, et al. Comparing caudal and penile nerve blockade using bupivacaine in hypospadias repair surgeries in children. Afr J Paediatr Surg. 2011;8(3):294-297. 